XLVIth Zakopane School of Physics, International Symposium Breaking Frontiers, Zakopane, Poland, May 16-21, 2011

\title{
From Magnetic Nanoparticles to Magnetoresistive Biosensors
}

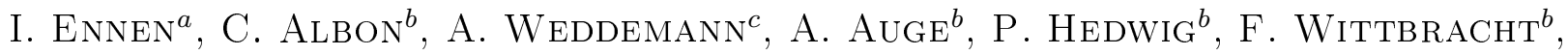 \\ A. Regtmeier ${ }^{b}$, D. Akemeier ${ }^{b}$, A. Dreyer ${ }^{d}$, M. Peter ${ }^{e}$, P. Jutzi ${ }^{d}$, J. Mattay $^{e}$, \\ N. Mitzel ${ }^{f}$, N. MiLl ${ }^{b}$ AND A. HÜtTEN ${ }^{b, *}$ \\ ${ }^{a}$ Institute of Solid State Physics, Vienna University of Technology, A-1040 Vienna, Austria
}

${ }^{b}$ Department of Physics, Thin Films and Physics of Nanostructures, Bielefeld University, 33615 Bielefeld, Germany

${ }^{c}$ Massachusetts Institute of Technology, RLE, LEES, 77 Massachusetts Ave, 02139 Cambridge, MA, USA

${ }^{d}$ Department of Chemistry, Organometallic Chemistry, Bielefeld University, 33615 Bielefeld, Germany

${ }^{e}$ Department of Chemistry, Organic Chemistry I, Bielefeld University, 33615 Bielefeld, Germany

${ }^{f}$ Department of Chemistry, Inorganic and Structural Chemistry, Bielefeld University, 33615 Bielefeld, Germany

This paper highlights recent advances in synthesis and magnetotransport properties of magnetic Co nanoparticles. It is shown that magnetic Co nanoparticles self-assembled in nanoparticular monolayers revealing giant magnetoresistance similar to granular systems but with additional features resulting from dipolar interactions between small domains of nanoparticles. A spin-valve with one magnetic Co nanoparticular electrode is employed as a model to demonstrate that individual magnetic moments of Co nanoparticles can be coupled to a magnetic Co layer which in turn offers tailoring of the resulting giant magnetoresistance characteristics. In addition, it is demonstrated that combining a magnetic on-off ratchet with magnetic tunneling junctions integrated in the ratchet introduces a new biosensor concept enabling: (1) simultaneous transporting and separating biomolecules, (2) dynamical biomolecule detection when passing magnetic tunneling junctions in a 1D arrangement. It is projected that this biosensor concept could be applied for viruses as well as for bacteria.

PACS: 85.70.-w, 85.75.-d, 75.75.Fk, 64.75.Yz, 87.85.fk

\section{Introduction}

The simultaneous discovery of the giant magnetoresistance (GMR) by Grünberg et al. [1] and Fert et al. [2] in 1988 was already based on two different GMR systems. While Grünberg was investigating $\mathrm{Fe} / \mathrm{Cr} / \mathrm{Fe}$ spin-valves, Fert was looking into the characteristics of $\{\mathrm{Fe} / \mathrm{Cr}\} \mathrm{N}$ multilayers so as to explore the origin of the GMR effect. Within in a very short time span thereafter both systems were driving the development of a new generation of read-heads (GMR spin-valves) and a new generation of sensors for automotive applications (GMR multilayers) [3]. Only about ten years after this discovery the potential of GMR sensors for the detection of magnetic beads was realized [4] and led to another technological avenue, the development of biosensors for life science applications.

Currently, magnetoresistive biosensors [5] use a new detection method for molecular recognition reactions based on a combination of magnetic markers and XMR sensors (where $\mathrm{X}=\mathrm{A}, \mathrm{G}, \mathrm{C}, \mathrm{T}$ ). Besides GMR-sensors also tunneling magnetoresistance (TMR) sensors are of great interest. Replacing the spacer layer in GMR spin- valves by a thin insulator such as $\mathrm{AlO}_{x}$ or $\mathrm{MgO}$ will lead to a TMR sensor. If this insulating layer is thin enough, e.g. about $2 \mathrm{~nm}$, electrons can tunnel from one ferromag-

\footnotetext{
* corresponding author; e-mail:

huetten@physik .uni-bielefeld.de
}

netic layer into the other - again a strictly quantum mechanical phenomenon. The tunneling probability is associated with the relative orientation of the magnetizations of the two adjacent ferromagnetic layers.

A parallel orientation yields a high tunnel current or an electrical state of low resistance whereas an antiparallel orientation is characterized by a low tunnel current or a state of high resistance. Like for GMR devices the TMR sensor can be switched between these two states of electrical resistance employing an external magnetic field.

As is shown in Fig. 1, the new detection method consists of superparamagnetic nanoparticles or beads which are specifically attached to a target molecule. The superparamagnetic nature of the nanoparticles or beads enables to switch on their magnetic stray fields by using an external magnetic field. Hence, the localization of the magnetic stray field by an embedded XMR sensor allows identifying the target molecule on or in close vicinity to the XMR sensor indicated by a drop in the electrical resistance.

The challenges of the development of such a combined tool for single molecule detection is fourfold: (1) the magnetic core of magnetic nanoparticles has to be stabilized by organic ligands so as to define their size distribution and simultaneously to preserve their magnetic property by preventing them from oxidation, (2) to functionalize the tail groups of the ligands such that biomolecules can easily be marked by these magnetic nanoparticles, (3) to design and realize XMR sensors which are capable of de- 


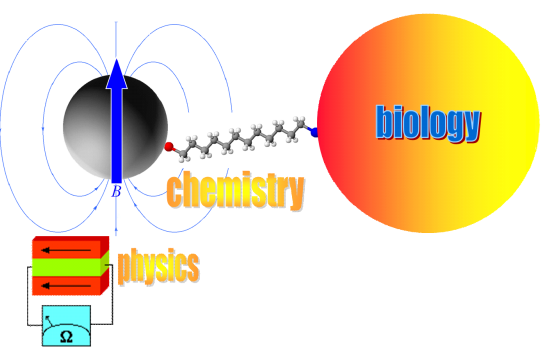

Fig. 1. Schematic presentation of a biosensor concept for single molecule detection.

tecting the magnetic stray field of magnetic nanoparticles enabling to count the number of magnetically labeled biomolecules covering the sensors surface and (4) to incorporate the sensors into a fluidic environment so as to ensure that all magnetically labeled biomolecules will pass by in low heights so as to ensure their binding onto the sensors surfaces in a static mode resulting in an interaction between their magnetic stray fields and the XMR sensors or allowing an interaction between the magnetic stray fields and the XMR sensors while passing by in a dynamic mode of analysis.

Within this framework our paper is focusing on GMR properties of magnetic Co-nanoparticles and on elaborating a new sensor concept consisting of magnetic tunneling junctions (MTJs) integrated in a magnetic on-off ratchet.

\section{Synthesis of magnetic Co-nanoparticles}

Thermolysis of magnetic nanoparticles was originally introduced by Puntes et al. [6, 7]. Tensides such as oleic acid, oleylamine or TOPO (tri-n-octylphosphine oxide) dissolved under inert conditions in an organic solvent and subsequently heated to reflux. The solvent temperature is adjusted to the decomposition temperature of an appropriate metalorganic precursors such as $\mathrm{Co}_{2}(\mathrm{CO})_{8}$ which starts to decompose when injected to the hot solvent and initiates the formation of nucleation seeds.

After formation, seeds absorb free metal atoms and continue to grow as is sketched in Fig. 2. The tensides act as stabilizers for the particles by forming a ligand shell around the metallic core. The particle growth dynamics can be explained in the frame of the LaMer and Dinegar model [8] which describes the growth process in two separate steps, see Fig. 2: above a critical concentration of free metal atoms, nucleation seeds are formed. Once the concentration drops below a critical threshold, the number of seeds remains constant and the existing seeds continue to grow.

The particle size can be controlled by a so-called successive particle synthesis [9] depicted in Fig. 3. During the growth process, repeated injection of precursor concentration below the nucleation threshold results in a continuous growth without producing any new seeds. The interaction between a tenside and the particle surface can occur in many ways and are mainly based on

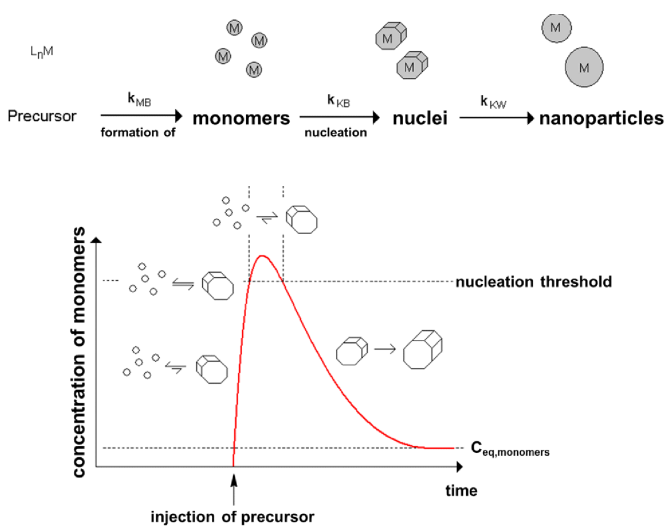

Fig. 2. Schematic illustration of LaMer's [8] nucleation and growth process so as to synthesis magnetic nanoparticles.

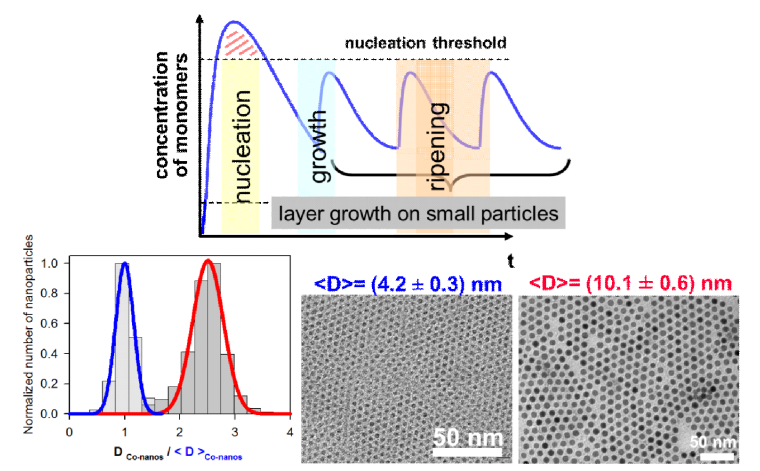

Fig. 3. The diameter of magnetic Co nanoparticles can be increased by successive addition of precursor solution employing, see upper sketch. The monomer concentration may not exceed the nucleation threshold. Resulting particle size distributions together with TEM bright field images are given below.

dipole-dipole, hydrogen bond- or Van der Waals interactions. They do usually not show covalent characteristics. The strength of the coupling between ligand and particle strongly affects the growth behavior of the metal cluster. The absorption of free metal atoms to the seed surface and, therefore, the continuation of growth is only possible at those areas where no complexes are present. A measure for the detachment of ligands is given by the dissociation constant $D_{\mathrm{e}}$. A small value of $D_{\mathrm{e}}$ corresponds to a hard to break bond between the metal surface and the ligand and, consequently, in reduced particle growth. The size of the dissociation constant may strongly vary, depending on the above mentioned binding affinities to different crystal planes.

Crystals with a simple cubic symmetry result in an isotropic value which entails spherical particles, see Fig. 4. However, if non-cubic crystal lattices are present, the dissociation constants may depend on the crystal plane and growth in specific directions is promoted $[6,10-12]$. 


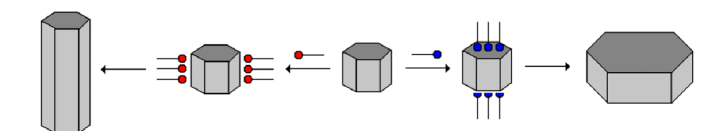

$\mathrm{Co}-$ dises

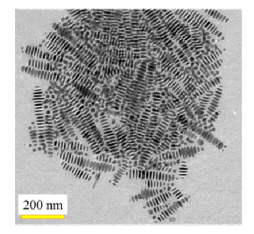

Co- cubes

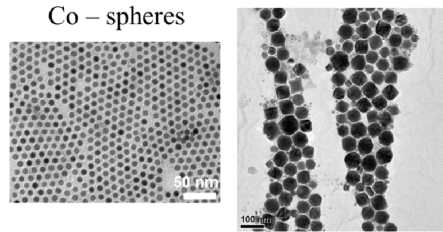

Fig. 4. TEM bright field images of magnetic Co-nanoparticles with different morphologies: discs, spheres and cubes, from left to right, below. Upper scheme sketches anisotropic nanoparticle growth due to ligand binding to specific crystal planes of nuclei.

\section{Nanoparticular GMR effect}

Due to this broad range of options in synthesis magnetic nanoparticles have been thoroughly studied during the last decades due to their many promising applications in chemical, physical and medical fields [13]. A common example is their employment in microfluidic devices. Due to their permanent magnetic moment, they can be controlled via external, inhomogeneous magnetic fields [14] and also be detected by magnetoresistive sensors [15] which allows for magnetobased monitoring of magnetically labeled biomolecules.

In this section we elaborate the potential of magnetic nanoparticle to serve as GMR sensors themselves. Diluted in a solvent after preparation these nanoparticles can be employed as magnetic India ink so as to be printed in form of monolayers onto different substrate. Using magnetic beads as a model system it has been demonstrated [16] that dipolar interaction between magnetic beads introduced by external magnetic rotational field will allow configuring these beads into chains below a critical rotation frequency. Overcoming the critical frequency will destroy these chains as a result of viscose sheer forces. The chain will break apart into two-dimensional small patches of beads which will agglomerate together in order to form highly ordered two-dimensional several $100 \mu \mathrm{m}$ large bead monolayers.

Meanwhile, we have transferred this method to magnetic Co-nanoparticles so as to prepare fairly large about $1 \mu \mathrm{m} \times 1 \mu \mathrm{m}$ nanoparticular array of hexagonal next neighbor coordination. Within such assemblies, magnetic nanoparticles themselves may act as magnetoresistive sensor devices. Surrounded by a non-magnetic matrix, various spin-dependent transport phenomena have been observed [17-21].

Contrary to formerly used metallurgic preparation techniques, nanoparticle fabrication by bottom-up chemical syntheses offer significant advantages. The systematic adjustment of the self-organization process by, e.g., the employment of ligands with different alkyl chain lengths, allows for the independent variation of the particle-matrix volume fraction and the inter-particle distances between the magnetic granules and, therefore, enables a systematic study of granular resistive effects.

The preparation sequence starting from a monolayer of magnetic Co-nanoparticle to reach a granular GMR structure is sketched in Fig. 5. One requirement is to remove the ligand shell and subsequently to electrically contact adjacent Co-nanoparticles by a thin metal, e.g. $\mathrm{Cu}$ - or Ru-overcoat of about $5 \mathrm{~nm}$ thickness so as to minimize electrical shunting. This procedure can be done in a UHV furnace by heating the nanoparticular monolayer at $400{ }^{\circ} \mathrm{C}$ for $5 \mathrm{~h}$ in a $95 \% \mathrm{~N}_{2}+5 \% \mathrm{H}_{2}$ gas atmosphere. Without breaking the vacuum, a thin $\mathrm{Cu}$-film is deposited to ensure electrically contact in between these nanoparticles.

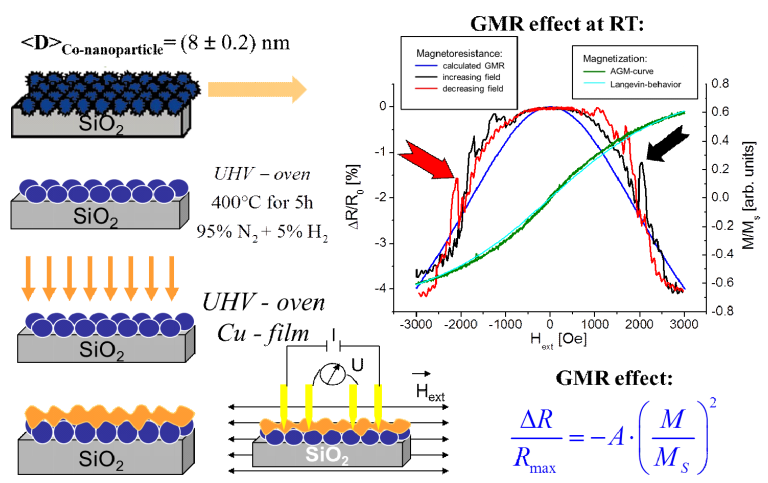

Fig. 5. GMR response of a monolayer consisting of $8 \mathrm{~nm}$ Co particles covered by a thin $\mathrm{Cu}$ film. Measurements were taken at room temperature with a sample current of $1 \mathrm{~mA}$ and an in-plane external magnetic field. In comparison to the prediction for non-interacting particles in blue, the experiments show additional features at field values symmetric to zero field as indicated by arrows. The blue line represents the calculated granular GMR effect.

A magnetotransport measurement using four-point probe geometry is given in Fig. 5 and was determined immediately after deposition of the overcoat in the same furnace without breaking the vacuum or immediately at ambient conditions so as to minimize oxidation. In comparison to the prediction of the GMR characteristic of non-interacting particles additional features showed up as sharp peaks at field values symmetric to zero field while sweeping the field from one direction to its opposite. Nevertheless, the resulting negative slope of the magnetoresistance curve for the current density and the external magnetic field in parallel when increasing the field clearly indicates a GMR behavior of the nanoparticular Co-monolayer. Finite element methods [22] have been applied in order to explore the origin of these additional peaks in the GMR-characteristic.

Preliminary results are summarized in Fig. 6. For a $10 \times 10$ Co nanoparticle array with a hexagonal next neighbor coordination peaks symmetric to zero field are resulting when assuming a distribution of small magne- 

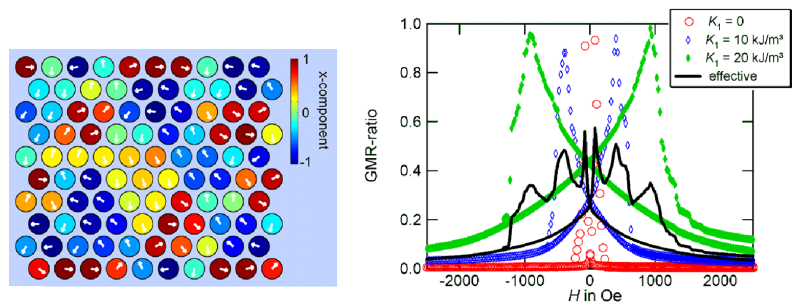

Fig. 6. Calculated magnetic moment distribution (left side) and GMR response (right side) of a monolayer consisting of $10 \times 10$ Co particles assuming a hexagonal next neighbor coordination and a distribution of a small magnetocrystalline anisotropies, $0 \mathrm{~kJ} / \mathrm{m}^{3}<K_{1}<$ $20 \mathrm{~kJ} / \mathrm{m}^{3}$. The resulting effective GMR characteristic in black shows similar features as being measured, compare with Fig. 5.

tocrystalline anisotropies in the range of $0 \mathrm{~kJ} / \mathrm{m}^{3}<K_{1}<$ $20 \mathrm{~kJ} / \mathrm{m}^{3}$. The peaks are probably due to the rotation of small nanoparticular domains towards the field direction. In equilibrium these domains are already present and are associated with purely dipolar interactions between the nanoparticles.

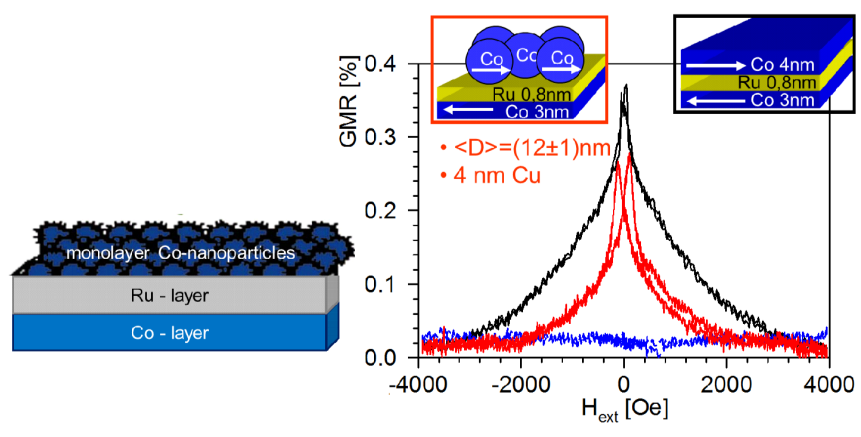

Fig. 7. Proof of concept of the idea (left side) that Co nanoparticles can be coupled to a Co layer via a $\mathrm{Ru}$ spacer layer coupling. For reference, the GMR characteristic (black curve, on right side) of three layers, $\mathrm{Co}_{3 \mathrm{~nm}} / \mathrm{Ru}_{0.8 \mathrm{~nm}} / \mathrm{Co}_{4 \mathrm{~nm}}$, measured at room temperature. The resulting nanoparticular GMR curve at room temperature (red curve) clearly indicates spin-valve character of $\mathrm{Co}_{3 \mathrm{~nm}} / \mathrm{Ru}_{0.8 \mathrm{~nm}} / \mathrm{Co}_{\mathrm{NP}}\langle 12 \mathrm{~nm}\rangle$.

With these findings the question arises whether a spin-valve can be realized by replacing one of the magnetic electrode layers by a nanoparticular monolayer as is pictured in Fig. 7. As a reference the layered spin-valve structure $\mathrm{Co}_{3 \mathrm{~nm}} / \mathrm{Ru}_{0.8 \mathrm{~nm}} / \mathrm{Co}_{4 \mathrm{~nm}}$ has been prepared and measured. The $0.8 \mathrm{~nm} \mathrm{Ru}$ spacer layer is associated with a first antiferromagnetic coupling maximum [23]. GMR effect amplitude of $0.36 \%$ at room temperature was achieved. It should be pointed out that no effort has been spent so as to optimize this value although it is clear that much larger effects can be realized in spin-valves [24]. The corresponding spin-valve structure with one nanoparticular Co-monolayer shows a similar GMR characteristic with an effect-amplitude of $0.28 \%$ at room temperature and clearly demonstrates the spin-valve character of $\mathrm{Co}_{3 \mathrm{~nm}} / \mathrm{Ru}_{0.8 \mathrm{~nm}} / \mathrm{Co}_{\mathrm{NP}}\langle 12 \mathrm{~nm}\rangle$. This strongly indicates that magnetic Co nanoparticles can be coupled to a magnetic Co layer utilizing the spacer layer coupling. From an application point of view this allows to tailor the GMR characteristics of nanoparticular GMR sensors which by the way show incredibly large sensitivities when used as biosensors as is shown in [25].

\section{Magnetic on-off ratchet: a new biosensor concept}

The fluidic environment which ensures an enhanced probability of binding labeled biomolecules onto XMR sensor surfaces is addressed here. One approach that can be used to transport biomolecules attached to magnetic beads is the on-off ratchet [26]. The combination of non-directional Brownian motion and the action of an asymmetric potential, which is periodically switched on and off may entail a directed transport without applying external forces.

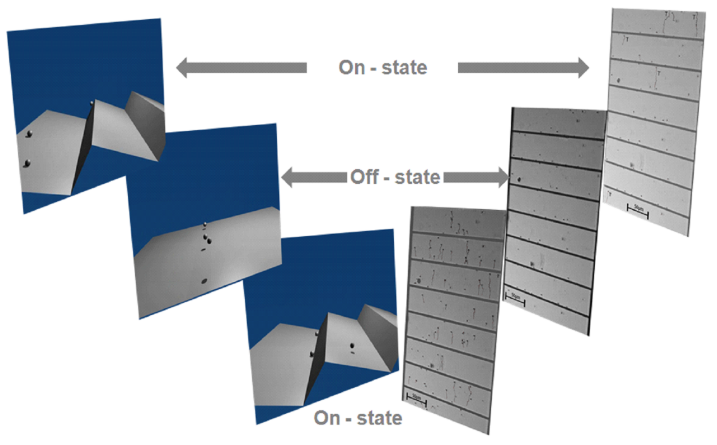

Fig. 8. Schematic illustration of the principle of a magnetic on-off ratchet on the left side. The magnetic potential acting in the off-state is pictured as an undulating landscape. In comparison, the experimental realization of the magnetic on-off ratchet on the right side is showing the corresponding bead movement. Clearly visible is the net flux of beads imaged as trace pattern. It arises from the asymmetric geometry of the magnetic potential visualized on the left hand side.

The on-off ratchet mechanism is illustrated in Fig. 8. The first state is the on-state, where beads move to their potential minimum. The second state is the off-state, where beads diffuse freely. Due to the asymmetry of the potential, which can experimentally be realized by a superposition of an assembly of spatially periodic conducting lines with a homogeneous magnetic field perpendicular to the conduction lines [27], the distance to the potential barrier on the steeper slope side is shorter than that on the gently inclined side. Thus the probability for beads to pass the potential barrier during the off-state on the steeper side is larger than that on the gently inclined side and hence a net flux of beads arises as is shown in Fig. 8 as well. Thus, this mechanism allows to intrinsically separating larger from smaller objects within the ratchet due to the reduced diffusivity of larger objects. 


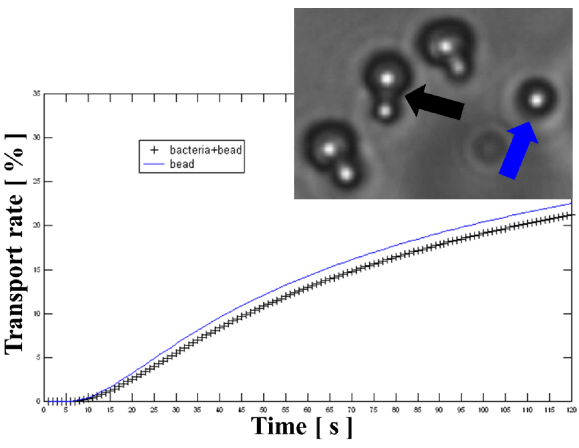

Fig. 9. Transport rates determined in the magnetic on-off ratchet for magnetic Chemagen M-PVA 1 beads (blue curve, marked with blue arrow in the inset). In comparison the resulting transport rate of magnetic Chemagen M-PVA 1 beads carrying Lawsonia bacteria (black curve, marked with a black arrow in the inset). The transport rate for the pure Chemagen M-PVA 1 beads is higher, as expected.

A proof of concept is given in Fig. 9 where the transport rate of Lawsonia bacteria bound to magnetic markers is compared to that of "naked" markers. Indeed, the latter are characterized by a larger transport rate. This result might trigger a new concept for biosensor which relays on the competition between increasing Brownian motion for decreasing object size and increasing inertia for increasing object size.

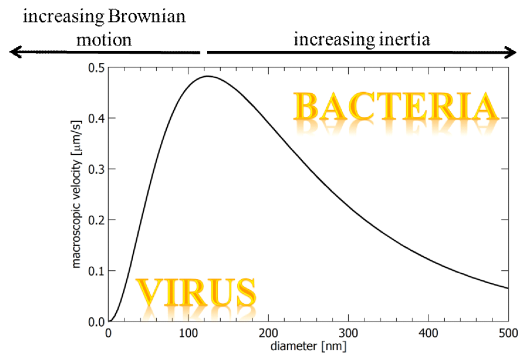

Fig. 10. Calculated macroscopic bead velocity for beads transported in the magnetic on-off ratchet as a function of their diameter.

An estimate of this potential is summarized in Fig. 10 where the macroscopic velocity is given as a function of this object diameter. The branch right of the maximum of this dependence could be associated with the separation of "naked" beads from those carrying bacteria whereas the branch left from the maximum could be reserved for the separation of smaller "naked" objects from those carrying viruses but is still experimentally to be proven.

\section{Dynamic bead detection employing MTJ arrays}

The complete innovation of the magnetic on-off ratchet becomes immediately visible when recognizing that ob- jects are highly localized about minimum positions of the magnetic potential during its on-state. Integrating highly sensitive magnetoresistive sensors right at these positions into the magnetic on-off ratchet will enable a dynamic detection process of magnetically labeled biomolecules. Possible candidates for those sensors are MTJs revealing large TMR-effect amplitudes at room temperature $[28,29]$. The stacking of such a MTJ is given in Fig. 11. The resulting TMR-effect amplitude is $117 \%$ at room temperature. Eight of this MTJs $2 \times 5 \mu \mathrm{m}^{2}$ in size has been patterned to a $1 \mathrm{D}$ array to demonstrate dynamical bead detection.
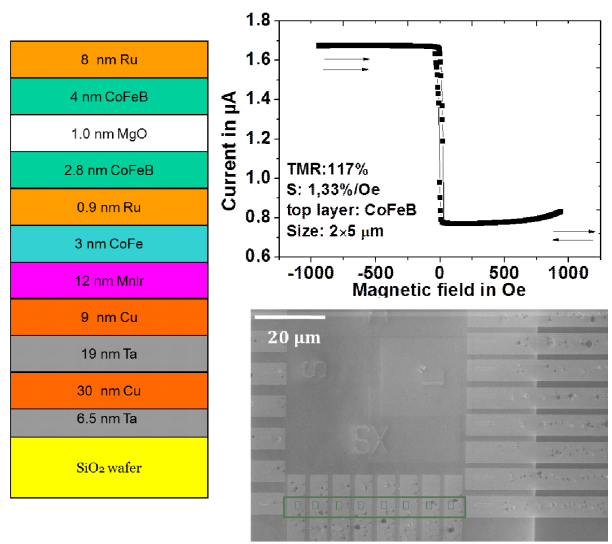

Fig. 11. MTJ layer stacking sequence (left side) with a room temperature TMR effect amplitude of $117 \%$. Eight of these MTJs have been patterned in a 1D array so as to demonstrate dynamical bead detection (SEM image on lower right side).

Employing external magnetic fields of $50 \mathrm{mT}$ perpendicular to the MTJs when magnetic beads are passing by in a microfluidic droplet of solvent is attracting the beads towards the sensor surface and initiates changes in the MTJ signal of about $10 \%$ for one bead only as is seen in Fig. 12.
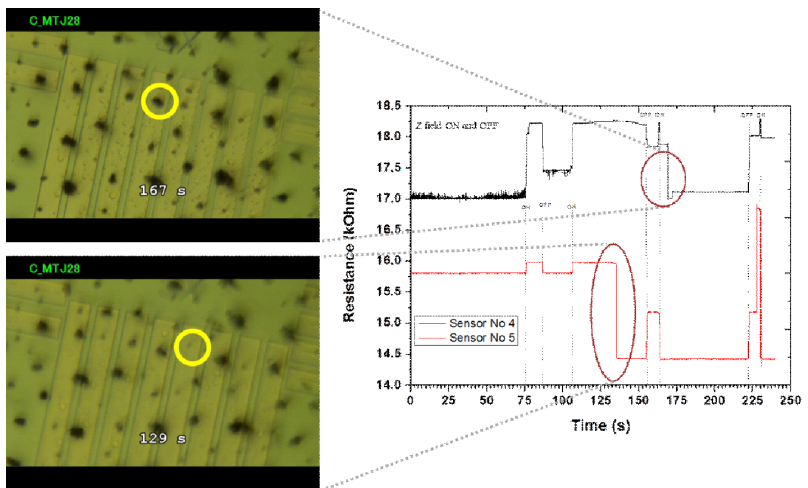

Fig. 12. Proof of concept of dynamical bead detection employing the MTJs of Fig. 11. A 9.6\% MTJ-signal change was measured while only one bead was passing one MTJ sensor. 
It is quite obvious to have a new biosensor concept at hand when combining a magnetic on-off ratchet with MTJ integrated in the ratchet. Hence this concept would provide: (1) simultaneous transporting and separating biomolecules, (2) dynamical biomolecule detection when passing MTJs. Moreover, it allows to completely transfer the molecular recognition to the bead surface and would enable molecular recognition in parallel when the beads size is matched to the size of individual biomolecules.

\section{Conclusions}

We have demonstrated that dipolar interaction between individual nanoparticles well separated will also lead to GMR. Introducing spacer layer coupling as an additional interaction in monolayers of Co nanoparticles enables nanoparticular spin-valve devices which could simply be printed in ASICs for future magnetotransport application. Furthermore, the potential of magnetic on-off ratchet in combination with MTJ integrated in the ratchet has been derived as a new concept for biosensors.

\section{Acknowledgments}

The authors affiliated with Bielefeld University would like to thank the SFB613 and the FOR 945 for financial support in the framework of the project $\mathrm{K} 3$ and 3, respectively, and the $\mathrm{BMBF}$ project MagRat (grant No. 16SV5048). Furthermore, Alexander Weddemann gratefully acknowledges his Feodor Lynen Fellowship from The Alexander von Humboldt foundation.

\section{References}

[1] G. Binasch, P. Grünberg, F. Saurenbach, W. Zinn, Phys. Rev. B 39, 4828 (1989).

[2] M.N. Baibich, J.M. Broto, A. Fert, F. Nguyen van Dau, F. Petroff, P. Eitenne, G. Creuzet, A. Friederich, J. Chazelas, Phys. Rev. Lett. 61, 2472 (1988).

[3] Magnetic Multilayers and Giant Magnetoresistance - Fundamentals and Industrial Applications, Ed. U. Hartmann, Springer Verlag, Berlin 2000.

[4] P.P. Freitas, R. Ferreira, S. Cardoso, F. Cardoso, J. Phys., Condens. Matter 19, 165221 (2007).

[5] D.R. Baselt, G.U. Lee, M. Natesan, S.W. Metzger, P.E. Sheehan, R.J. Colton, Biosensors Bioelectron. 13, 731 (1998).

[6] V.F. Puntes, K.M. Krishnan, A.P. Alivisatos, Science 291, 2115 (2001).

[7] V.F. Puntes, K.M. Krishnan, A.P. Alivisatos, Appl. Phys. Lett. 78, 2187 (2001).

[8] V.K. LaMer, R.H. Dinegar, J. Am. Chem. Soc. 72, 4847 (1950).
[9] A. Hütten, D. Sudfeld, I. Ennen, G. Reiss, W. Hachmann, U. Heinzmann, K. Wojczykowski, P. Jutzi, W. Saikaly, G. Thomas, J. Biotechnol. 112, 47 (2004).

[10] V.F. Puntes, D. Zanchet, C. Erdonmez, A.P. Alivisatos, J. Am. Chem. Soc. 124, 12874 (2002).

[11] X. Peng, L. Manna, W. Yang, W.J. Wickham, E. Scher, A. Kadavanich, A.P. Alivisatos, Nature 404, 59 (2000).

[12] K. Soulantica, A. Maisonnat, F. Senocq, M.C. Fromen, M.J. Casanove, B. Chaudret, Angew. Chem. 113, 3071 (2001).

[13] G. Reiss, A. Hütten, Nat. Mater. 4, 725 (2005).

[14] N. Pamme, Lab Chip 6, 24 (2006).

[15] J. Loureiro, R. Ferreira, S. Cardoso, P.P. Freitas, J. Germano, C. Fermon, G. Arrias, M. Pannetier Lecoeur, F. Rivadulla, J. Rivas, Appl. Phys. Lett. 95, 034104 (2009).

[16] A. Weddemann, F. Wittbracht, B. Eickenberg, A. Hütten, Langmuir 26, 19225 (2010).

[17] S. Wang, F.J. Yue, D. Wu, F.M. Zhang, W. Zhong, Y.W. Du, Appl. Phys. Lett. 94, 012507 (2009).

[18] H. Zeng, C.T. Black, R.L. Sandstrom, P.M. Rice, C.B. Murray, S. Sun, Phys. Rev. B 73, 020402 (2006).

[19] T. Wen, D. Liu, C.K. Luscombe, K.M. Krishnan, Appl. Phys. Lett. 95, 082509 (2009).

[20] R.P. Tan, J. Carrey, C. Desvaux, J. Grisolia, P. Renaud, B. Chaudret, M. Respaud, Phys. Rev. Lett. 99, 176805 (2007).

[21] R.P. Tan, J. Carrey, M. Respaud, C. Desvaux, P. Renaud, B. Chaudret, J. Magn. Magn. Mater. 320, L55 (2008).

[22] A. Weddemann, A. Auge, F. Wittbracht, D. Kappe, A. Hütten, J. Magn. Magn. Mater. 322, 643 (2010).

[23] P.J.H. Bloemen, H.W. van Kesteren, H.J.M. Swagten, W.J.M. de Jonge, Phys. Rev. B 50, 13505 (1994).

[24] A. Hütten, T. Hempel, S. Heitmann, G. Reiss, Phys. Status Solidi A 189, 327 (2002).

[25] A. Weddemann, I. Ennen, A. Regtmeier, C. Albon, A. Wolff, K. Eckstädt, N. Mill, M. Peter, J. Mattay, C. Plattner, N. Sewald, A. Hütten, Beilstein J. Nanotechnol. 1, 75 (2010).

[26] P. Hänggi, F. Marchesoni, Rev. Mod. Phys. 81, 387 (2009).

[27] A. Auge, A. Weddemann, F. Wittbracht, A. Hütten, Appl. Phys. Lett. 94, 183507 (2009).

[28] C. Albon, A. Weddemann, A. Auge, K. Rott, A. Hütten, Appl. Phys. Lett. 95, 023101 (2009).

[29] C. Albon, A. Weddemann, A. Auge, D. Meißner, K. Rott, A. Hütten, Appl. Phys. Lett. 95, 163106 (2009). 